\title{
Isótopos Estables, Dieta de Camélidos y Diferenciación Social (Valle De Ambato, Catamarca, Argentina, Siglos VI-XI D.C.)
}

\author{
Stable Isotopes, Camelids Diet and Social Differentiation (Ambato Valley, Catamarca, \\ Argentina, VI-XI Centuries AD) \\ Mariana Dantas, Germán Figueroa, Andrés Laguens y Andrés Izeta ${ }^{i}$
}

RESUMEN

En este trabajo se discute el modo en que se organizó la producción ganadera en el Valle de Ambato, Catamarca, entre los siglos VI y XI d.C., a partir de nuevos análisis isotópicos de $\delta^{13} \mathrm{C}$ realizados sobre restos de camélidos. Los resultados alcanzados, sumados a los obtenidos previamente en otros trabajos, permiten plantear la existencia de dos formas de sustento de los camélidos: una de movilidad extensa y alimentación muy variada vs. otra de movilidad restringida y alimentación controlada. Esta última habría sido uno de los elementos fundamentales que habría conformado un sistema de producción agropastoril integrado, que articulaba la cría de plantas y camélidos en un mismo espacio y con una misma infraestructura.

Palabras Clave: Camélidos Sudamericanos, Isótopos Estables, Aguada De Ambato.

\section{ABSTRACT}

In this paper we discuss how livestock production was organized in Ambato Valley, Catamarca, between the $\mathrm{VI}$ and $\mathrm{XI}$ centuries $\mathrm{AD}$, based on new $\delta^{13} \mathrm{C}$ isotopic analyzes performed on camelid remains. The results achieved, together with those previously obtained in other studies, suggest the existence of two forms of livelihood of camelids: one of extensive mobility and varied food vs. other of restricted mobility and controlled feeding. The latter would have been one of the key elements that have conformed an integrated agropastoral productive system, that articulated camelid and plant breeding in the same space and with the same infrastructure.

Key Words: South American Camelids, Stable Isotopes, Aguada Culture.

i Instituto de Antropología de Córdoba (IDACOR), CONICET y Museo de Antropología, Universidad Nacional de Córdoba, Hipólito Yrigoyen 174, 5000 Córdoba, Argentina. Correo-e: dantasmariana@hotmail.com, g.gfigueroa@ hotmail.com, laguens@ffyh.unc.edu.ar, andresizeta@gmail. com.

Recibido: 05-12-2012 Revisado: 04-06-2013 Aceptado: 01-03-2014 


\section{INTRODUCCIÓN}

Desde aproximadamente el 300 d.C. hasta el 1.000 d.C. se desarrolló en el Valle de Ambato, Provincia de Catamarca, Argentina, una nueva forma de vida caracterizada por la cultura Aguada, que estuvo basada en la intensificación en la explotación de los recursos, un marcado incremento de la población, junto con diversificación de roles sociales, una incipiente especialización artesanal y marcadas desigualdades sociales y políticas (Laguens 2006). Para estas sociedades los camélidos tuvieron gran importancia. Así, por ejemplo, en lo económico, fueron un elemento significativo para la subsistencia, constituyendo la principal fuente de proteínas dentro de la dieta de estos grupos. Al mismo tiempo, los restos de esta familia presentan un patrón de distribución diferencial en los espacios habitados, algo que pudo haber obedecido a la existencia de distinciones sociales entre las diferentes unidades domésticas del valle (Dantas 2010). Conjuntamente, estos animales participaron en ámbitos no sólo económicos, sino también sociales e ideológicos, tales como contextos fundacionales de muros y estructuras de combustión, o contextos rituales singulares (Laguens y Bonnin 2005).

A partir de este marco general, buscamos profundizar nuestros conocimientos sobre las modalidades de obtención y manejo de los camélidos, centrándonos especialmente en la producción ganadera. Los resultados conseguidos hasta el momento nos permitieron observar que los camélidos presentan valores enriquecidos de $\delta^{13} \mathrm{C}$, lo cual se vincula con una ingesta elevada de plantas $\mathrm{C}_{4}$. Esto resulta un hecho totalmente novedoso para la región, que apunta a que los animales tuvieron una alimentación controlada compuesta por el consumo de maíz en altas proporciones, a partir de los restos agrícolas (Izeta et al. 20l0). Esto llevó a considerar que entre los siglos $\mathrm{VI}$ y XI d.C., en el valle existió una estrategia productiva agropastoril dentro de la cual la cría de camélidos se habría caracterizado por la restricción en su alimentación, con pastoreo a campo abierto muy limitado y una movilidad reducida en torno a corrales entre los campos de cultivo, cerca de las aldeas residenciales del fondo del valle (Figueroa et al. 20I0).

Considerando esta información, un interrogante que surgió fue si esta práctica de manejo fue generalizada en esta sociedad o sólo una forma de producción diferenciada en distintos sectores de la sociedad. Entonces, con el propósito de indagar sobre estos aspectos, nos propusimos replicar los análisis $\delta^{13} \mathrm{C}$ en restos faunísticos procedentes de diversas clases de sitos, que corresponden a distintas unidades sociales -tales como sitios grandes de elite, o unidades residenciales pequeñas- buscando obtener datos que aporten nuevos elementos a la comprensión de esta problemática.

\section{MATERIALESY MÉTODOS}

Los materiales considerados en este trabajo proceden de los sitios Martínez I (MI), Martínez 2 (M2), Martínez 3 (M3), Piedras Blancas (PB), El Altillo (EA) y Recinto 4 (R4). Los cuatro primeros se localizan en el sector deprimido del valle, en tanto que EA se emplaza en el piedemonte y R4 sobre la ladera occidental (Figura I). MI, M2, PB y R4 son sitios que presentan ocupaciones Aguada, EA tiene ocupaciones pre-Aguada y M3 ambas.

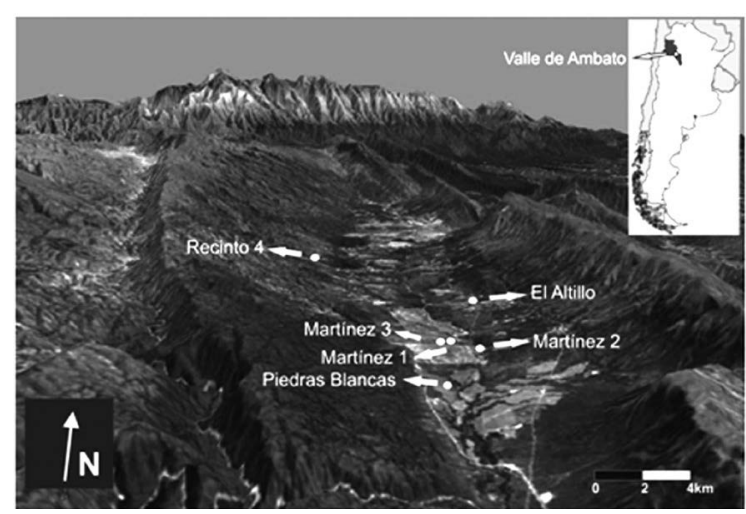

Figura I: Mapa del Valle de Ambato con ubicación de sitios. Figure I:Ambato Valley map with location of the sites.

EA es un sitio monticular al aire libre ubicado sobre una elevación natural del terreno, rellenado con materiales cerámicos, líticos, faunísticos, botánicos y restos humanos (Assandri 2007) (I.900 $\pm 70 \mathrm{AP}$, ramas carbonizadas, LP474, Bonnin y Laguens 1997). El sitio MI consta de una unidad-habitación y de un montículo basurero, fue ocupado por grupos domésticos de reducido número que desarrollaron allí diversas actividades artesanales (Assandri 199I) (I.770 \pm 90 AP, carbón, LP46I, Bonnin y Laguens 1997). M2 presenta dos sectores de 
habitaciones separadas por un patio central, rodeado por un muro perimetral (Juez 1991) (990 $\pm 70 \mathrm{AP}$, carbón, LP; I.690 \pm 80 AP, carbón, LP444; Bonnin y Laguens 1997, Laguens 2006). En tanto, M3 fue caracterizado como un sitio al aire libre monticular, aislado, compuesto por una acumulación de diversos materiales arqueológicos (Assandri 2007). Ávila y Herrero (199I) identificaron dos momentos en la conformación del sitio, correspondiendo los niveles inferiores a ocupaciones pre-Aguada (Componente II, I.700 \pm 60 AP, carbón, LP553, Bonnin y Laguens 1997) y los superiores a Aguada (Componente I, $1.458 \pm 44$ AP, hueso de camélido, AA93889).

Por su parte, PB es un sitio que presenta un montículo y un sector con construcciones, el cual fue definido como una residencia de elite de grandes dimensiones (Assandri 2007). En base a los fechados radiocarbónicos, se puede situar el período de ocupación del sitio entre el 600 y 1.000 d.C. (Laguens 2006). Finalmente, R4 es una estructura de planta rectangular con muro de piedra doble, que se haya inserta en un sistema de terrazas de cultivo y cuya funcionalidad fue interpretada como corral (Dantas y Figueroa 2009) (1.312 \pm 43 AP, hueso de camélido, AA93890).

Con el propósito de cumplimentar con los objetivos planteados anteriormente se realizaron análisis de isótopos estables de carbono y nitrógeno a 35 muestras procedentes de restos de camélidos de MI, M2 y M3. A estos resultados sumamos los datos de $\delta^{13} \mathrm{C}$ obtenidos con anterioridad en PB, EA, M3 y R4', en su mayoría ya publicados en trabajos previos (Izeta et al. 2009, 20I0). Las muestras seleccionadas corresponden a elementos del esqueleto apendicular de camélidos que presentaban buen aspecto visual y se encontraban en buen estado de conservación (no estaban quemados, meteorizados, ni con depositación química, etc.). Asimismo, se priorizaron aquellos especímenes que fueron clasificados dentro de la categoría llama a través de los análisis osteométricos $^{2} y$, con posterioridad, se incluyeron otros elementos de camélidos de tamaño grande.

Los análisis isotópicos fueron realizados en el Environmental Isotope Laboratory, University of Arizona. Los valores de $\delta^{13} \mathrm{C}$ y $\delta^{15} \mathrm{~N}$ son expresados en partes por mil (\%o) en relación a los estándares internacionales V-PDB y AIR, respectivamente. La precisión es mejor que $\pm 0,10$ para $\delta^{13} \mathrm{C}$ y $\pm 0,2$ para $\delta^{15} \mathrm{~N}($ Is), basada en estándares internos. Tanto los valores de $\delta^{15} \mathrm{~N}$ y $\delta^{13} \mathrm{C}$, así como el contenido de carbono y nitrógeno se midieron en un espectrómetro de masas de flujo continuo (Finnigan Delta PlusXL) acoplado a un analizador elemental (Costech). Se calculó la relación C:N para medir el impacto de procesos diagenéticos que pudieron reducir el contenido de colágeno de los huesos. La mayor parte de las muestras se encuentra dentro del rango aceptable, entre 2,7 y 3,6 (Pate 1994) o entre 2,6 y 3,4 (Schoeninger et al. 1989), salvo dos ejemplares de $M 2$ que tienen valores de 3,8 y 4,2 (Tabla I). En consecuencia, estos últimos no serán incluidos en la discusión de los resultados debido a que reflejan una señal isotópica que podría no ser primaria.

En cuanto a la ecología isotópica del valle, Izeta et al. (2009) registraron las especies vegetales con capacidad forrajera, así como su distribución y vía fotosintética. De este modo, pudieron observar la presencia de especies con patrones fotosintéticos $\mathrm{C}_{3}$ y $\mathrm{C}_{4}$, las que se distribuyen indistintamente en los diferentes pisos vegetales del valle (i.e. bosque serrano, pastizal-arbustal y pastizal de altura), sin presentar ningún tipo de correlación con la altitud.

\section{RESULTADOSY DISCUSION}

En la Tabla I se presentan los resultados del análisis de las muestras de MI, M2 y M3 seleccionadas para esta investigación. Estos datos son analizados en forma conjunta con los 27 valores ya disponibles para PB, EA, M3 y R4 (Tabla 2).

En MI, M2 y ambos componentes de M3 los valores de $\delta^{13} \mathrm{C}$ presentan una considerable variabilidad, oscilando entre $-19,9 \%$ y $-11,0 \%$, con un desvío estándar de entre I,87 y 2,70 (Tabla 3). Estos datos indican que estos animales se habrían estado alimentando en diversos grados con una dieta mixta de plantas con patrones fotosintéticos $\mathrm{C}_{3}$ y $\mathrm{C}_{4}$. Del mismo modo, si bien hay una considerable diversidad entre los distintos individuos muestreados, la mayor parte de los mismos presenta por sitio una media que varía ente $-15,0 \%$ y $-12,5 \%$ 。 $(\mathrm{M} 3 \mathrm{Cll}=$ $12,5 ; \mathrm{M} 3 \mathrm{Cl}=13,9 ; \mathrm{Ml}=15,1 ; \mathrm{M} 2=14,4)$, acentuando la tendencia a una dieta con porcentajes semejantes de plantas $\mathrm{C}_{3}$ y $\mathrm{C}_{4}$. Estos resultados se asemejan a los obtenidos con anterioridad en EA, donde también se 
identificaron valores entre $-17,1 \%$ y $-11,8 \%$, con una media de $-14,0 \%$ y una desviación estándar de 2,2I. En MI, el ejemplar MI/22, constituye un caso único, ya que presenta una señal sumamente negativa que se asemeja a la registrada en animales que habitan en zonas ecológicas con un porcentaje mayor de plantas $\mathrm{C}_{3}$, como la Puna (Fernández y Panarello 1999-200I, Samec 20II, Yacobaccio et al. 2009).

En contraste con estos sitios, los especímenes de PB presentan una señal isotópica más enriquecida, ya que el valor más bajo registrado es de $-13,1 \%$ ○ el más alto de $-9,5 \%$, con una media de $-1 \mathrm{I}, 3 \%$. Además, la variabilidad existente entre los individuos es mucho menor, como lo refleja la desviación estándar de I, I0 (Tabla 3). Estos resultados denotan que los camélidos procedentes de este sitio recibieron una alimentación compuesta por mayores proporciones de plantas con patrón fotosintético $\mathrm{C}_{4}$, la cual a su vez fue muy restringida o controlada. Una interpretación similar fue alcanzada por Svoboda (2010) a partir de los resultados obtenidos en un centro ceremonial del valle, el sitio Iglesia de los Indios o La Rinconada, datado para la misma época. La única muestra obtenida del R4 presenta un valor de $-12,2 \%$, que indica un consumo con mayores proporciones de plantas $\mathrm{C}_{4}$, si se considera lo señalado por Tykot (2006) que una dieta constituida por una mezcla de $50 \%$ de plantas $\mathrm{C}_{3}$ y $50 \%$ de $\mathrm{C}_{4}$ produce un valor de $\delta^{13} \mathrm{C}$ de @-14,5\%.

\begin{tabular}{|c|c|c|c|c|c|}
\hline Muestra & Grupo & $\delta \mathbf{I 3 C} \%$ & $\delta \mathbf{I 5 N} \%$ & $\mathbf{C} / \mathbf{N}$ & Laboratorio \\
\hline \multicolumn{6}{|c|}{ Martínez 3 Componente I } \\
\hline $\mathrm{M} 3 \mathrm{Cl} / 02$ & Lama sp. & $-11,5$ & 5,4 & 2,9 & A \\
\hline $\mathrm{M} 3 \mathrm{Cl} / 03$ & Lama sp. & $-15,3$ & 5,2 & 2,9 & A \\
\hline $\mathrm{M} 3 \mathrm{Cl} / 04$ & Lama sp. & $-14,8$ & 3,9 & 2,9 & A \\
\hline $\mathrm{M} 3 \mathrm{Cl} / 09$ & Llama & $-12,6$ & 4,9 & 3,0 & A \\
\hline $\mathrm{M} 3 \mathrm{Cl} / \mathrm{IO}$ & Llama & $-14,0$ & 4,2 & 2,8 & A \\
\hline $\mathrm{M} 3 \mathrm{Cl} / \mathrm{II}$ & Lama sp. & $-16,8$ & 4,5 & 2,9 & A \\
\hline $\mathrm{M} 3 \mathrm{Cl} / \mathrm{I} 2$ & Lama sp. & $-11,9$ & 5,5 & 2,9 & $A$ \\
\hline $\mathrm{M} 3 \mathrm{Cl} / \mathrm{I} 3$ & Lama sp. & $-17,1$ & 4,6 & 2,8 & A \\
\hline $\mathrm{M} 3 \mathrm{Cl} / \mathrm{I} 6$ & Lama sp. & $-12,0$ & 5,7 & 2,9 & A \\
\hline $\mathrm{M} 3 \mathrm{Cl} / 20$ & Lama sp. & $-13,7$ & 3,9 & 2,9 & A \\
\hline \multicolumn{6}{|c|}{ Martínez 3 Componente II } \\
\hline $\mathrm{M} 3 \mathrm{Cll} / 0 \mathrm{I}$ & Lama sp. & $-13,0$ & 3,9 & 2,8 & A \\
\hline $\mathrm{M} 3 \mathrm{ClI} / 05$ & Llama & $-11,3$ & 4,3 & 2,9 & A \\
\hline $\mathrm{M} 3 \mathrm{ClI} / 06$ & Lama sp. & $-11,0$ & 4,7 & 2,9 & A \\
\hline $\mathrm{M} 3 \mathrm{ClI} / 07$ & Llama & $-11, I$ & 5,0 & 2,9 & A \\
\hline $\mathrm{M} 3 \mathrm{ClI} / 08$ & Lama sp. & $-11,9$ & 5,4 & 3,0 & A \\
\hline $\mathrm{M} 3 \mathrm{CII} / \mathrm{I} 4$ & Lama sp. & $-12,9$ & 8,8 & 2,9 & A \\
\hline $\mathrm{M} 3 \mathrm{CII} / \mathrm{I5}$ & Lama sp. & $-14,2$ & 3,9 & 2,9 & A \\
\hline $\mathrm{M} 3 \mathrm{ClI} / \mathrm{I7}$ & Lama sp. & $-11,3$ & 5,2 & 2,9 & A \\
\hline $\mathrm{M} 3 \mathrm{ClI} / \mathrm{I8}$ & Lama sp. & $-11,2$ & 5,2 & 2,9 & A \\
\hline $\mathrm{M} 3 \mathrm{ClI} / \mathrm{I} 9$ & Lama sp. & $-16,8$ & 5,5 & 2,9 & A \\
\hline \multicolumn{6}{|c|}{ Martínez I } \\
\hline $\mathrm{MI} / 2 \mathrm{I}$ & Lama sp. & $-11,6$ & 5,9 & 2,8 & A \\
\hline $\mathrm{Ml} / 22$ & Lama sp. & $-19,9$ & 2,4 & 2,9 & $A$ \\
\hline $\mathrm{MI} / 23$ & Lama sp. & $-13,6$ & 4,1 & 3,0 & A \\
\hline $\mathrm{Ml} / 25$ & Lama sp. & $-16,2$ & 5,8 & 3,6 & A \\
\hline $\mathrm{Ml} / 26$ & Lama sp. & $-15,5$ & 4,4 & 3,1 & $A$ \\
\hline $\mathrm{MI} / 27$ & Lama sp. & $-14,8$ & 4,5 & 2,8 & $A$ \\
\hline $\mathrm{MI} / 28$ & Llama & $-11,8$ & 4,5 & 3,0 & $A$ \\
\hline $\mathrm{Ml} / 29$ & Llama & $-16,4$ & 3,8 & 2,9 & A \\
\hline
\end{tabular}




\begin{tabular}{|l|c|c|c|c|c|}
\hline Martínez 2 & & & & & \\
\hline M2/24 & Lama sp. & $-13,7$ & 6,0 & 2,9 & A \\
\hline M2/30 & Llama & $-17, I$ & 7,1 & 3,0 & A \\
\hline M2/3I & Lama sp. & $-1 \mathbf{6 , 5}$ & $\mathbf{8 , 5}$ & $\mathbf{4 , 2}$ & A \\
\hline M2/32 & Lama sp. & $-16, I$ & 4,5 & 3,0 & $\mathrm{~A}$ \\
\hline M2/33 & Lama sp. & $-1 \mathbf{6 , I}$ & $\mathbf{7 , 0}$ & $\mathbf{3 , 8}$ & $\mathbf{A}$ \\
\hline$M 2 / 34$ & Lama sp. & $-12,5$ & 3,5 & 2,8 & $\mathrm{~A}$ \\
\hline$M 2 / 35$ & Lama sp. & $-12,5$ & 9,8 & 3,0 & $\mathrm{~A}$ \\
\hline
\end{tabular}

Tabla 1: Resultados de análisis de isótopos de carbono y nitrógeno.

Table 1: Carbon and nitrogen isotope analysis results.

Las tendencias descriptas entre los conjuntos analizados resultan aún más notorias al graficarlas en la Figura 2, ya que como puede advertirse los materiales procedentes de PB claramente se diferencian del resto de los sitios, presentando un agrupamiento mucho más positivo y concentrado.

\begin{tabular}{|c|c|c|c|}
\hline Muestra & Grupo & $\delta I 3 C(\% \circ)$ & Laboratorio \\
\hline \multicolumn{4}{|l|}{ Piedras Blancas } \\
\hline PB99 CI5 39 & Llama & $-9,5$ & AIE \\
\hline PB00 RH NI04 4I & Llama/guanaco & $-9,9$ & AIE \\
\hline Piedras Blancas & Lama sp. & $-10,1$ & AIE \\
\hline PB96 CI NI2 C'0I & Llama & $-10,2$ & AIE \\
\hline PB99 C56 N3 300 & Llama/guanaco & $-10,3$ & AIE \\
\hline PB00 RH NI07 62-B & Llama & $-10,4$ & AIE \\
\hline PB99 TM CE NI 021 & Llama & $-10,7$ & AIE \\
\hline PB99 C8 NI9 h.36 & Llama & $-10,8$ & AIE \\
\hline PB00 RH NI06 I7 & Llama/guanaco & $-11,0$ & AIE \\
\hline PB00 RH NI07 I5-B & Llama & $-11,9$ & AIE \\
\hline PB96 CI NII A`00 & Llama & $-11,9$ & AIE \\
\hline PB96 CINI2 C'79 & Llama & $-12,0$ & AIE \\
\hline PB99 C56 N3 303 & Llama & $-12,7$ & AIE \\
\hline PB96 C2 N22 A63 & Llama & $-12,7$ & AlE \\
\hline PB99 CI5 44 & Llama/guanaco & $-13, \mid$ & AIE \\
\hline PB Rec. H Estruct. I & Lama sp. & $-11,2$ & AA \\
\hline PB Rec. F & Lama sp. & $-11,5$ & AA \\
\hline PB Patio G & Lama sp. & $-12,7$ & AA \\
\hline \multicolumn{4}{|l|}{ El Altillo } \\
\hline El Altillo & Lama sp. & $-16,2$ & AIE \\
\hline EA S2 NI0600 & Llama & $-11,8$ & AIE \\
\hline EA S2 NI 3844 & Llama & $-13,1$ & AIE \\
\hline EA S2 NI384I & Llama & $-15,5$ & AIE \\
\hline EA S2 NI 3826 & Llama & $-17,1$ & AIE \\
\hline EA S2 NI6046 & Llama & $-12,1$ & AIE \\
\hline EA S2 NI6040 & Llama & $-12,2$ & AIE \\
\hline \multicolumn{4}{|c|}{ Martínez 3 Componente I } \\
\hline M3 & Llama & $-13,4$ & AA \\
\hline \multicolumn{4}{|l|}{ Recinto 4} \\
\hline Recinto 4 & Lama sp. & $-12,2$ & AA \\
\hline
\end{tabular}

Tabla 2: Valores de isótopos de carbono previos.

Table 2: Previous carbon isotope values. 


\begin{tabular}{|c|c|c|c|c|c|c|}
\hline & $\mathrm{N}$ & Mínima & Máxima & Media & Mediana & $\mathrm{DE}$ \\
\hline EA & 7 & $-17,1$ & $-11,8$ & $-14,0$ & $-13,1$ & 2,21 \\
\hline $\mathrm{M} 3 \mathrm{Cll}$ & 10 & $-16,8$ & $-11,0$ & $-12,5$ & $-11,6$ & $\mathrm{I}, 87$ \\
\hline $\mathrm{M} 3 \mathrm{Cl}$ & II & $-17,1$ & $-11,5$ & $-13,9$ & $-13,7$ & 1,92 \\
\hline MI & 8 & $-19,9$ & $-11,6$ & $-15,0$ & $-15,1$ & 2,70 \\
\hline M2 & 5 & $-17,1$ & $-12,5$ & $-14,4$ & $-13,7$ & 2,12 \\
\hline PB & 18 & $-|3|$, & $-9,5$ & $-11,3$ & $-|1|$, & 1,10 \\
\hline
\end{tabular}

Tabla 3: Estadística descriptiva de las muestras discriminada por sitio.

Table 3: Descriptive statistics of the samples discriminated per site.

Por otro lado es necesario considerar que si bien todos los especímenes analizados en $\mathrm{MI}$, M2 y M3 corresponden a camélidos grandes, sólo en pocos casos fue posible asignarlos a la categoría llama a través de los análisis osteométricos, por lo que cabe la posibilidad de que dentro de las muestras estudiadas haya especímenes de guanaco. No obstante, los análisis de isótopos estables de carbono realizados a especímenes procedentes de PB que mediante estudios osteométricos se agruparon con los guanacos del NOA, arrojaron valores semejantes a los registrados para el grupo llamas. Esta situación llevó a considerar la presencia en el valle de animales domésticos de menor porte, esto es, llamas pequeñas, corrigiendo la asignación osteométrica obtenida inicialmente (Dantas 2012). Por lo que es factible que las muestras examinadas en este caso también correspondan en su mayoría a animales domésticos, especialmente si tomamos en cuenta que los especímenes que efectivamente pudieron asignarse a la categoría llamas presentan valores isotópicos de $\delta^{13} \mathrm{C}$ muy variados (v.g. $-11,1 \%$, $-16,4 \%$, $-17,1 \% \circ)$, sin presentar un patrón diferenciado con respecto al resto de la muestra.

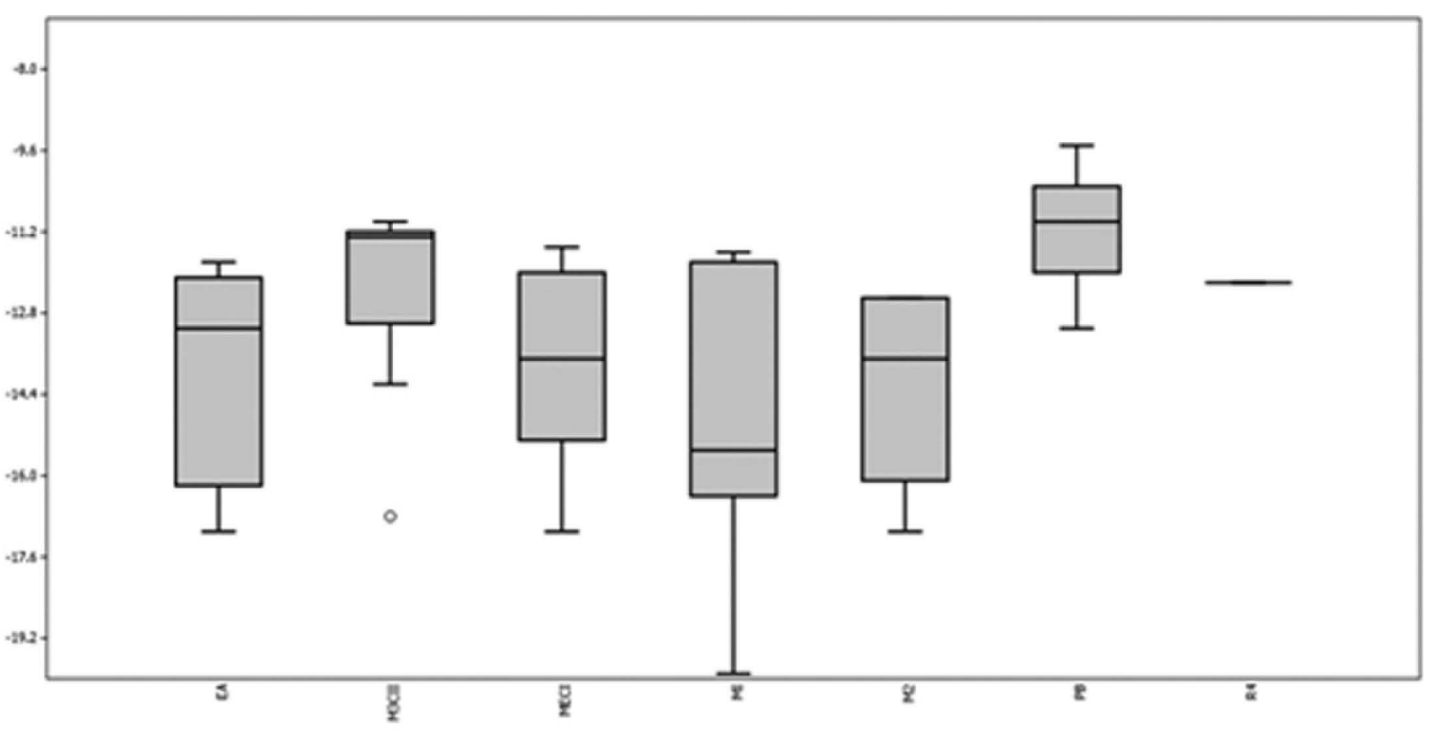

Figura 2: Resultados de los análisis de isótopos de carbono.

Figure 2: Carbon isotope analysis results. 


\section{CONSIDERACIONES FINALES}

En base a los resultados obtenidos se puede observar que durante el lapso de ocupación Aguada los camélidos estaban alimentándose con dos patrones claramente diferenciados. Por un lado, el que presentan los individuos procedentes de $\mathrm{PB}$, con altas proporciones de plantas $\mathrm{C}_{4}$, dentro de las que predominó el maíz ${ }^{3} y$, por el otro, el registrado en los sitios restantes, con una dieta que incluyó una mayor diversidad de plantas $\mathrm{C}_{3}$ y $\mathrm{C}_{4}$ concordante con los valores de la ecología isotópica local.

De este modo, podemos considerar que en el Valle de Ambato, entre los siglos VI y XI d.C., nos encontramos con dos formas distintas de manejo de los animales. En primer lugar, una en que las llamas tuvieron una movilidad mayormente restringida en torno a los corrales localizados entre los campos de cultivo, practicando en una forma limitada el pastoreo en campos abiertos y recibiendo, de este modo, una alimentación controlada en base a maíz, ya sea a partir de los restos agrícolas o en frutos. En segundo lugar, una práctica de manejo de los animales con pastoreo a campo abierto y con una movilidad más amplia.

Esta última habría sido una práctica ya existente durante momentos pre-Aguada ${ }^{4}$ y que proveyó de animales a la mayor parte de los sitios estudiados. En tanto, la primera habría sido una intensificación propia de la ocupación más tardía, cuya producción habría tenido una distribución circunscrita a los sitios de elite del valle. Este panorama sugiere que existió una distribución y/o acceso diferenciado de los animales criados bajo cada una de las estrategias productivas.

\section{NOTAS}

I La determinación de $\delta^{13} \mathrm{C}$ se llevó a cabo en el Laboratorio de Isótopos Ambientales, Instituto de Geocronología y Geología Isotópica,Argentina (AIE) y el Arizona Radiocarbon AMS Facility, University of Arizona (AA).

2 Ver Dantas (2010) para mayor detalle de los estudios osteométicos específicos.

3 En este caso, resulta necesario considerar que, los trabajos realizados desde el año 2005 hasta la actualidad, posibilitaron observar que entre los 1.100 y 1.600 msnm, se ubica un sector destinado a la producción animal y vegetal, de aproximadamente $800 \mathrm{ha}$, el cual incluye infraestructura agrícola y ganadera (i.e.corrales, viviendas, terrazas de cultivo, obras de riego y de almacenamiento de agua) que comparte el mismo espacio. Del sedimento recobrado de algunas de estas estructuras agrícolas se efectuaron análisis de silicofitolitos y almidones, pudiéndose determinar que en las mismas se practicó el cultivo exclusivo de maíz y que gran parte de esta producción habría sido transportada a los sitios residenciales emplazados en el fondo del valle (Figueroa 2012, 20I3; Zucol et al. 20I2).

${ }^{4}$ Cabe señalar que en el sitio $M 3$, en momentos pre-Aguada también había ya una práctica de alimentación controlada, mediante especies $C_{4}$, además de la combinación de $C_{3} y$ $\mathrm{C}_{4}$ (Figura 2). Esta situación nos lleva a considerar dos posibles explicaciones: primero, dado que M3 que no se trataría de un sitio residencial, ya que es un montículo con un patrón de depositación particular de objetos que apunta más a prácticas especiales, vinculadas con procesos de construcción de memorias (Gastaldi 2010) y con el ceremonial doméstico (Ávila y Herrero 1991), no es extraño que se hayan depositado restos de animales tratados diferencialmente; una segunda hipótesis se vincula con la posibilidad de que esta práctica agropastoril ya haya comenzado a implementarse hacia finales de las ocupaciones pre-Aguada y que, posteriormente, con la implementación del modo de vida Aguada se haya desarrollado más sistemáticamente, a una escala mayor, al mismo tiempo que orientando su distribución y consumo sólo a ciertos sectores de la sociedad.

\section{BIBLIOGRAFÍA}

Assandri, S.B. 1991. "Primeros resultados de la excavación en el sitio de Martínez I". Publicaciones del CIFFYH, Arqueología 46: 53-86.

2007. Procesos de complejización social y organización espacial en el Valle de Ambato, Catamarca, Argentina. Universidad Internacional de Andalucía, Sede Iberoamericana Santa María de la Rábida: La Rábida. URI: http://hdl.handle.net/10334/62.

Ávila, A. y R. Herrero. 1991. "Secuencia estratigráfica del sitio arqueológico Martínez 3, Dpto. Ambato, Catamarca". Publicaciones del CIFFYH, Arqueología 46: I7-52.

Bonnin, M.I. y A.G. Laguens. 1997. "Evaluación de series de fechados radiocarbónicos del valle de Ambato, Catamarca". Publicaciones del CIFFYH, Arqueología 48: 65-I0I.

Dantas, M. 2010. Arqueología de los animales y procesos de diferenciación social en el valle de Ambato, Catamarca,Argentina. Tesis para optar al grado de Doctor en Historia, Facultad de Filosofía y Humanidades, Universidad Nacional de Córdoba, Córdoba.

2012. "El rol de los animales en contextos sociales no igualitarios: el caso del Valle de Ambato, Catamarca, Argentina". Revista del Museo de Antropología 5(2): 259-268.

Dantas, M. y G.G. Figueroa. 2009.“Terrazas y corrales como espacios integrados de producción agro-pastoril en el Valle de Ambato, Catamarca, Argentina (s. VI-XI d.C.)". Relaciones de la Sociedad Argentina de Antropología XXXIV: 343-350.

Fernández, J. y H.O. Panarello. 1999-200I."Isótopos del carbono en la dieta de herbívoros y carnívoros de los Andes jujeños". Xama I2-14: 7I-85.

Figueroa, G.G. 2012. Pircas, llamas y maíz. Un estudio arqueológico y etnoarqueológico de sistemas de producción de montaña en el Noroeste Argentino. Editorial Académica Española, Saarbrücken. 
2013. "Estrategias productivas en Aguada de Ambato (Catamarca, Argentina)". Relaciones de la Sociedad Argentina de Antropología XXXVIII: III-I35.

Figueroa, G.G., M. Dantas y A. Laguens. 2010 . "Practicas agropastoriles e innovaciones en la producción de plantas y animales en los Andes del Sur. El Valle de Ambato, Argentina, primer milenio d.C." International Journal of South American Archaeology 7: 6-13.

Gastaldi, M. 2010. Cultura material, construcción de identidades y transformaciones sociales en el Valle de Ambato durante el primer milenio d. C. Tesis para optar al grado de Doctor en Ciencias Naturales, Facultad de Ciencias Naturales, Universidad Nacional de La Plata, La Plata. URI: http://sedici.unlp.edu.ar/ handle/I0915/5316

Izeta, A.D., A.G. Laguens, M.B. Marconetto y M.C. Scattolin. 2009. "Camelid handling in the meridional Andes during the first millennium AD: a preliminary approach using stable isotopes. International Journal of Osteoarchaeology 19(2): 204-I4.

Izeta, A.D., M. Dantas, M.G. Srur, M.B. Marconetto y A.G. Laguens. 20 I0. "Isótopos estables y manejo alimentario de camélidos durante el primer milenio A.D. en el Valle de Ambato (Noroeste Argentino)". En La arqueometría en Argentina y Latinoamérica, editado por S. Bertolino, R. Cattáneo y A. Izeta, Pp. 237-242. Editorial de la Facultad de Filosofía y Humanidades, Universidad Nacional de Córdoba, Córdoba.

Juez, M. S. 1991. "Unidad arqueológica Rodeo Grande, Valle de Ambato: excavación en el sitio Martínez 2". Publicaciones del CIFFYH,Arqueología 46: 87-II 0.

Laguens, A.G. 2006."Continuidad y ruptura en procesos de diferenciación social en comunidades aldeanas del Valle de Ambato, Catamarca, Argentina (s. IV - X d.C.)". Chungará 38(2): 207-18.
Laguens, A.G. y M. Bonnin. 2005."Recursos materiales y desigualdad social en la arqueología de Ambato- Catamarca". En La cultura de La Aguada y sus expresiones regionales: trabajos presentados en la V Mesa Redonda La Cultura de La Aguada y su Dispersión, Pp. 23-33. EUDELAR, La Rioja.

Pate, F.D. 1994. "Bone Chemistry and Paleodiet". Journal of Archaeological Method and Theory I (2): I6I-209.

Samec, C.T. 20I I. Perspectiva isotópica sobre la alimentación de camélidos domésticos y silvestres de la Puna Jujeña: Construyendo un marco de referencia para estudios arqueológicos. Tesis de Licenciatura, Facultad de Filosofía y Letras, Universidad de Buenos Aires, Buenos Aires.

Shoeninger, M.J., K. M. Moore, M.L. Murray y J.D. Kingston. 1989. "Detection of bone preservation in archaeological and fossil samples". Applied Goechesmistry 4: 28I292.

Svoboda, A. 2010. Análisis de los conjuntos arqueofaunísticos de un núcleo residencial de La Rinconada (Valle de Ambato, Catamarca). Tesis de Licenciatura, Facultad de Filosofía y Letras, Universidad de Buenos Aires, Buenos Aires.

Tykot, R.H. 2006. "Isotope Analyses and the Histories of Maize". En Histories of Maize in Mesoamerica. Multidisciplinary Approaches, editado por J.E. Staller, R.H. Tykot y B.F. Benz, pp. I3 I142. Academic Press, San Diego.

Yacobaccio, H.D., M.R. Morales y C.T. Samec. 2009. "Towards an Isotopic Ecology of Herbivory in the Puna Ecosystem: New Results and Patterns on Lama glama". International Journal of Osteoarchaeology 19: 144-155.

Zucol, A.F., G.G. Figueroa y M.M. Colobig. 2012. "Estudio de microrestos silíceos en sistemas de aterrazamiento del primer milenio d.C. en el Valle de Ambato (Andes del Sur), Catamarca, Argentina". Intersecciones en Antropología 13: 163-179. 